\title{
Antiviral, cyototoxic and antimicrobial activities of anthraquinones isolated from the roots of Morinda elliptica
}

\begin{abstract}
2-Formyl-1-hydroxyanthraquinone, along with ten other known anthraquinones (1-hydroxy2-methylanthraquinone, nordamnacanthal, damnacanthal, lucidin-?-methyl ether, rubiadin, rubiadin-1-methyl ether, soranjidiol, morindone, morindone-5-methyl ether and alizarin-1methyl ether), isolated from the roots of Morinda elliptica, were assayed for anti-HIV, cytotoxic and antimicrobial activites. Only damnacanthal showed moderate activity against HIV. It was cytotoxic towards the MCF-7 (breast carcinoma) and CEM-SS (T-lymphoblastic leukaemia) cell line. Nordamnacanthal was very cytotoxic against the CEM-SS cell lines. Other anthraquinones that showed strong cytotoxicity towards the cell lines tested were lucidin-?-methyl ether (CEM-SS and MCF-7) and rubiadin (CEM-SS). Three anthraquinones viz., nordamnacanthal, damnacanthal and morindone, were found to have strong antimicrobial activity.
\end{abstract}

Keyword: Anthraquinones; Antimicrobial; Antiviral; Cytotoxic; Morinda elliptica 